\title{
Anode Column Behavior of Long Vertical Air Arcs at Atmospheric Pressure
}

\author{
DON J. LATHAM
}

\begin{abstract}
A theoretical model for the unit cross section of a 1-atm unbounded arc was used to investigate arc behavior. The electrical source was taken as a dc voltage and resistance in series. Because the simulated arc is not radially bounded, the arc $V-I$ characteristic is found to be time varying and dependent on the source values. The results of exercising the model also show that the central temperature of the arc is maintained in the neighborhood of $7000 \mathrm{~K}$ and that changes in the source values can only raise this temperature for times on the order of $100 \mu \mathrm{s}$, approximately the time for diffusion of a temperature perturbation from the core of the arc to its periphery. The growth of the arc into ambient air is governed by the diffusivity of the ambient air, and is considerably slower.
\end{abstract}

\section{INTRODUCTION}

$\mathrm{T}$ HE LIGHTNING continuing current is a naturally occurring arc discharge, on the order of $2.5 \mathrm{~km}$ long, known to be an effective ignition source for wildland fires. The arc is initiated by a lightning return stroke spark discharge. As an aid in understanding the continuing current, and in developing a laboratory surrogate discharge for fuel ignition studies, we have use for a model for the positive column of the long vertical free arc.

The behavior of such arcs at atmospheric pressure was investigated by King [1] and Strom [12] experimentally, and decay modeled by Uman and Voshall [3]. Latham [4] presented a quasi-steady-state model. Matsumura et al. [5], using a model by Hermann [6], show experimentally that models such as that of [4] may be applied to timedependent behavior of wall-stabilized arcs. Similar models have been used by Lowke [7] and Lee [8]. Jones and Freeman [9] give an analytic solution. These models, excepting [4], have neglected either radiation or joule heating, or have a confining cold boundary. Free arcs effectively confined by electrode effects and axial flow present a different modeling problem from that considered here. In presenting the results from our earlier model [4], we gave temperature profiles for a unit cross section of a free boundary arc but the time behavior of the model arc was not reported, and the arc characteristic not discussed. In this paper, we report the results of applying an improved version of the model to the simulation of long arcs with realistic electrical sources.

Manuscript received June 21, 1985; revised November 26, 1985.

The author is with the USDA Forest Service Intermountain Research Station, Intermountain Fire Sciences Laboratory, Missoula, MT 59807.

IEEE Log Number 8608118.

\section{THE MODEL}

The model, as in [4], is a unit cross section of a radially symmetric optically thin arc, unbounded in radius, at constant pressure (1 atm), with joule heating input locally balanced by thermal diffusion, mass transport, heating, and radiation loss, as pointed out by Maecker [10]. The arc is in local thermodynamic equilibrium. Mass movement due to temperature changes is taken into account via the equation of continuity, but due to the constant pressure assumption, the momentum equation is not used. We consider that the arc is sufficiently long that electrode effects, including generation of axial flow, are negligible. Magnetic effects have been neglected, although they may be important for currents as low as $100 \mathrm{~A}$ [11] for the free arcs under consideration. We have used a radiation loss calculation by Adams [12].

Due to the absence of a heat-sink wall, the anode column of the long free arc must continually expand by conduction and mass flow if there is no axial flow within the arc. If the arc is very long, axial flow must be very small or absent, except close to the electrodes. The presence of the free boundary demands that radial velocity cannot be assumed zero everywhere, as in the steady-state wall-stabilized dc arc [8]. Because the velocity has no radius at which it is constrained, other than to be zero at the center of the arc, there is no characteristic radius useful in calculation. There can be at best only a quasi-steady-state solution to the set of plasma equations. As we shall show, the characteristic of such an arc is not unique; the arc behavior is dependent on the electrical source.

The electric current source for the present model is a Thevenin equivalent, that is, a voltage (which in general may be time varying) in series with an impedance; lowfrequency complex sources may always be reduced to this combination. For investigation of the time-varying behavior of a dc arc, we use a dc source voltage and a pure resistance as the impedance. Because the model arc is treated in terms of a unit length, the source voltage is given as an electric field $E_{s}$ and the source resistance as a resistance per unit length $R_{s}$. The source short-circuit current $I_{s}$ is that current which would flow if the arc were an electrical short. The arc electrode drops are subsumed in the source. Model exercises were also done under a constant current constraint, and some under a constant electric field. 
An outline of the model equations used and the method of solution are given in the Appendix. A more complete description is given in [4].

\section{Results of Exercising the Model}

The environment for a model run consists of specification of an initial temperature profile and choice of an electrical source and its parameters. The amount of output generated by variation of the initial conditions and parameters becomes large very quickly. As we want to discuss modeling of expanding arcs initiated by sparks, we restrict the presentation of results to those for a single initial temperature profile, as shown in Fig. 1, with a central temperature of $10300 \mathrm{~K}$. After the first $100 \mu \mathrm{s}$ or so, the behavior of the model channel is insensitive to the shape of this profile. Data points were generated at 5-ms intervals. Exercises of the model with shorter reporting intervals demonstrated that the initial 5-ms interval is ample "settling time" to substantially reduce the effect of the initial transient.

A set of radial temperature profiles with time as a parameter is shown in Fig. 1. The same general features were evident in the temperature profiles for the other Thevenin equivalent source exercises, as well as those for constant current sources. The model arc has a core in which the temperature decays with time, and an outer region growing in size at a decreasing rate. The radial growth continues even if the source is interrupted or the source resistance increased. Results from interrupting the model source are very close to those of Uman and Voshall [3], except that our model central temperature decays slightly more rapidly because we included radiation loss. Note that the $5-\mathrm{ms}$ profile is quite reasonable for a confined arc of 1.5-2-cm radius [6].

Radial growth for model sources is shown in Fig. 2. Because the outer boundary of the model arc does not have a characteristic dimension, such as a wall diameter, we have chosen the radius within which 99.99 percent of the electric current flows (for brevity, hereafter called the radius). This radius turned out to be very close to the radius within which 99.99 percent of the radiation was emitted. The growth rate of the model arc is clearly dependent on the electrical source characteristics.

The best index to the radial growth rate seems to be the source short-circuit current. That is, as the short-circuit current capability of the source increases, so does the radial growth rate. The details of the trajectory of the growth curve are dependent on the source field $E_{s}$, as well as the short-circuit current value. Constant current radial growth rates are very similar to those for Thevenin sources, again with differing details in the growth trajectory. A given Thevenin source curve tends to be asymptotic to the growth curve for a constant current model for the associated $I_{s}$. The radial growth of decaying channels was found to be very slow by comparison.

Attempts to parameterize the arc radius as a function of time and source characteristics failed. In particular, the

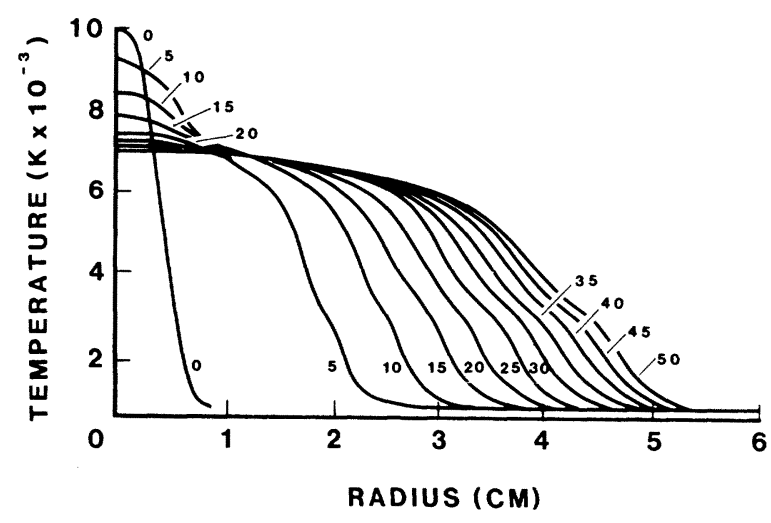

Fig. 1. Radial temperature structure of a model arc as a function of time at 5-ms intervals. $E_{s}=24 \mathrm{~V} / \mathrm{cm} ; R_{s}=0.1 \Omega / \mathrm{cm}$.

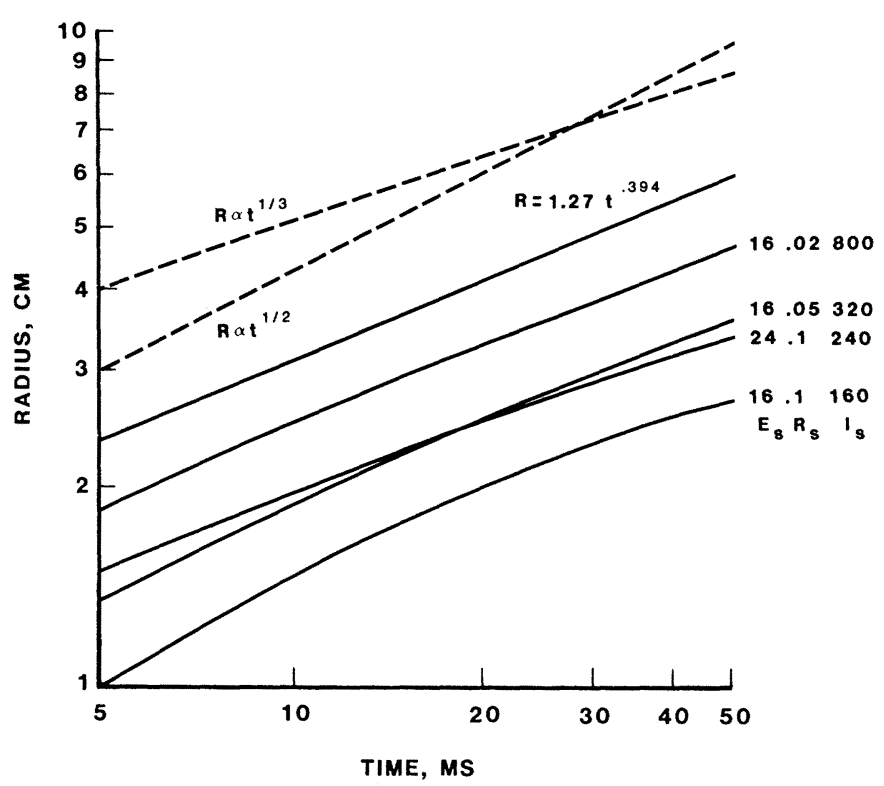

Fig. 2. Electrical radius (see text) as a function of time for various Thevenin sources. $E_{s}$ is in volts per centimeter; $R_{s}$ is in ohms per centimeter; and $I_{s}$ is in amperes.

possibility that there might be a similarity solution in a variable $V=r t^{-n}$ ( $r$ is the radius and $t$, time) was extensively checked, both against model equations and data from model runs, with negative results. This validated our initial suspicion that the equations are simply too nonlinear to admit of such a solution, and that the free boundary of this problem could not be treated in the same way as that of a simpler problem such as the temperature field evolution with a long wire or tube as a heat source. Note that solutions do lie near $r \alpha t^{1 / 3}$ and $r \alpha t^{1 / 2}$, at least for $t<$ $50 \mathrm{~ms}$ of model time (Fig. 2).

Fig. 3 presents the center temperature decay as a function of time and source parameters. As with the radial growth, no simple approximation for the temperature decay was possible. There seem to be two or three different decay modes, again most conveniently labeled by $I_{s}$. Up to $I_{s} \cong 200 \mathrm{~A}$, the initial decay (for time less than $5 \mathrm{~ms}$ ) is extremely rapid, followed by a slight increase and then a slow decrease. For $I_{s}$ between 200 and $500 \mathrm{~A}$, there is 


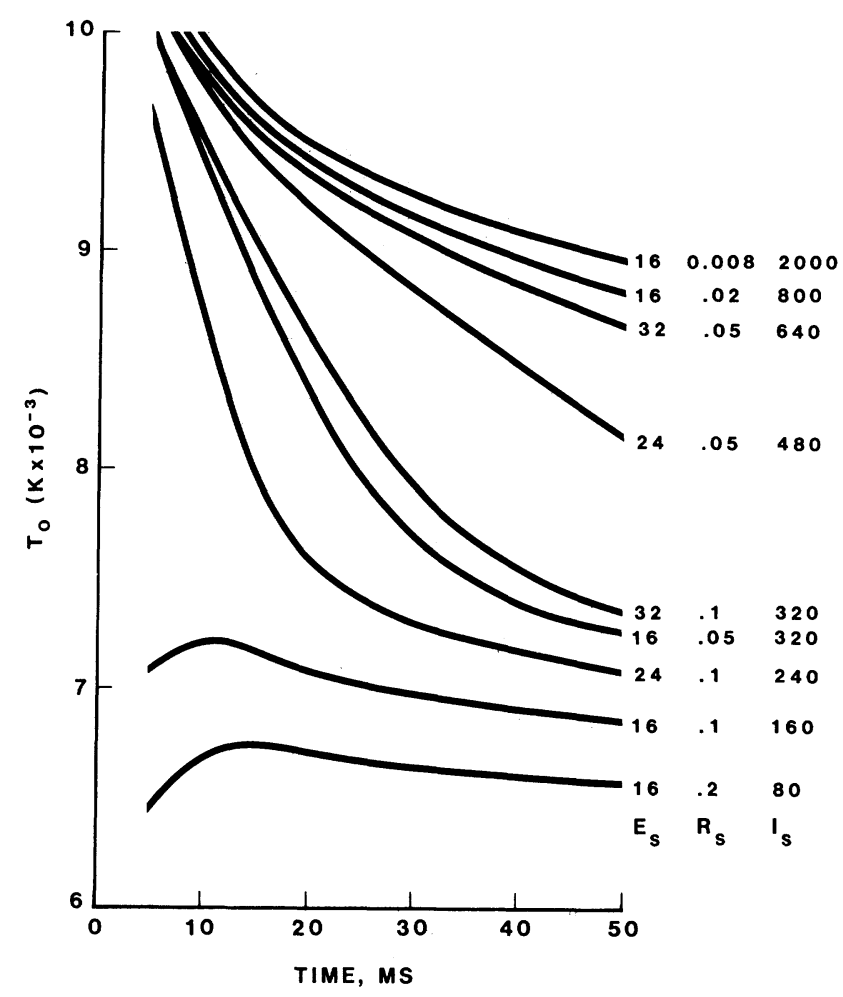

Fig. 3. Temperature at the center of the arc model as a function of time for various Thevenin sources. (Units as in Fig. 2.)

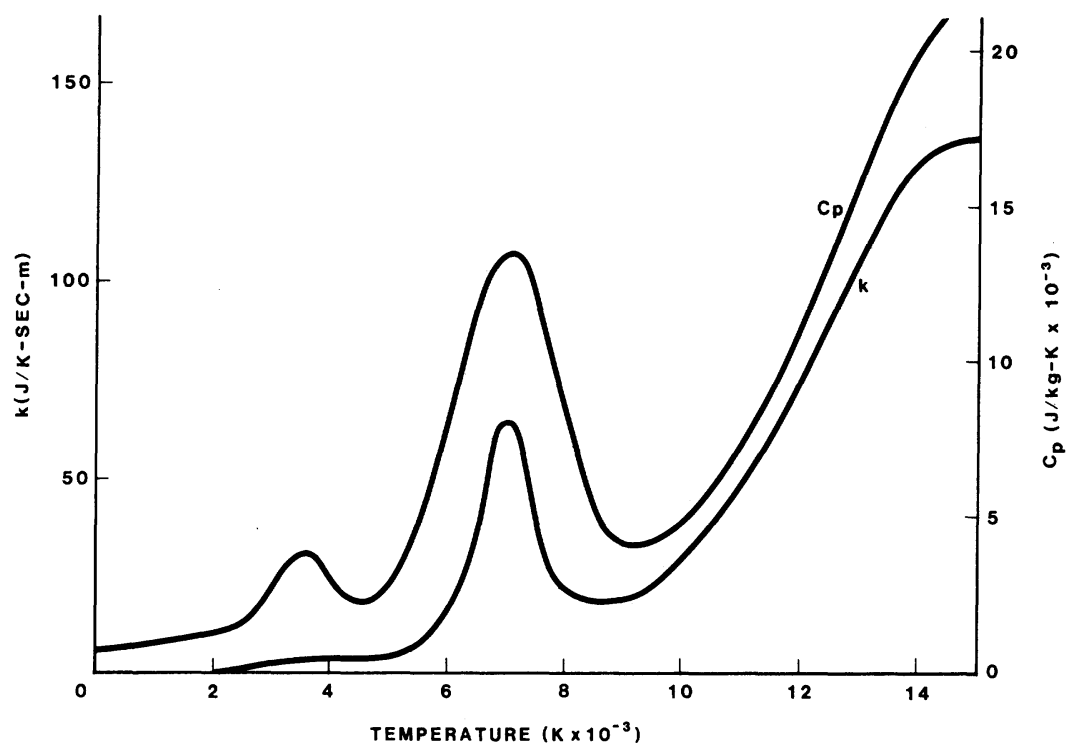

Fig. 4. Specific heat at constant pressure $C_{p}$ and thermal conductivity $k$ of air at one atmosphere pressure versus temperature.

a decrease to a temperature near $7250 \mathrm{~K}$ within $30-40 \mathrm{~ms}$. For $I_{s}$ larger than $500 \mathrm{~A}$, the temperature decrease rate seems to be significantly slower than for currents less than that value. In general, however, the larger the short-circuit current, the slower the central temperature decay.

The behavior of the temperature trajectories may be due to the thermal conductivity and specific heat characteristics (Fig. 4). The equations simulating the arc seem to operate so as to keep the central conductivity and specific heat as large as possible, i.e., away from the minimum near $8500 \mathrm{~K}$. We have neither devised a way to test this mathematically nor can we offer a physical reason, other than perhaps the maximum entropy (Steenbeck) principle [14].

Comparison of decaying arcs with corresponding output from other models shows that the initial decay of the central temperature is somewhat faster than that for a free arc model with no radiation [3], but slower than the decay of a wall-stabilized arc model with radiation [6].

The part of the overall energy balance played by radiation is depicted in Fig. 5, in which the radiation efficiency is plotted as a function of time for Thevenin sources. The 


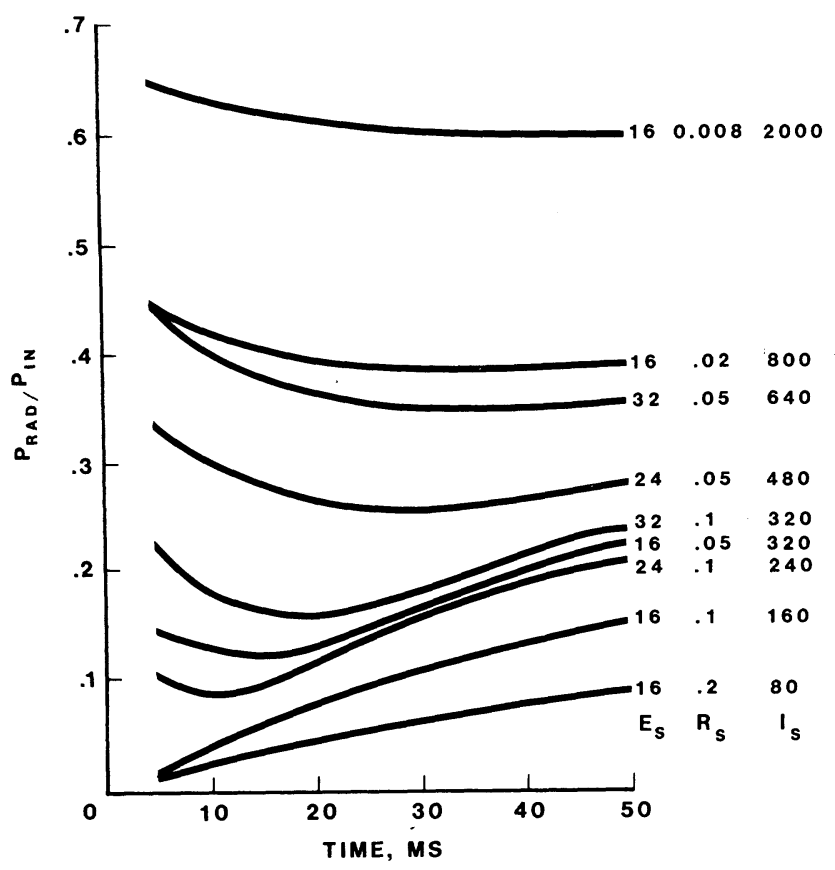

Fig. 5. Radiation efficiency $P_{\text {rad }} / P_{\text {in }}$ as a function of time for various Thevenin sources. (Units as in Fig. 2.)

radiation efficiency was found by integrating the radiation radially, under the assumption that the gas is optically thin [13]. The value obtained was divided by the input power (current/electric-field product) for the same time. Once again, the short-circuit current seems to be the best index to behavior. The trajectories of the curves look different, but a close examination shows commonality. The efficiency at first decreases, then begins to increase with time.

The time for which minimum radiation occurs increases as $I_{s}$ increases, and the variation in amplitude is smaller. This is explained by a look at Figs. 1 and 3. For small model time, the radiation, proportional approximately to $T^{9}$ [15], drops off rapidly due to cooling. Later, however, channel expansion provides a larger volume, albeit at a lower temperature, the rate of cooling slows, and radiation increases. There is a large range of efficiencies, from 10 to 70 percent, and further exercises of the model might extend this range.

With Fig. 6, we turn to the electrical behavior of the model. This figure presents the model arc channel current normalized to the short-circuit current, as a function of modeled time. The curves can now be ordered by the source voltage, subordered by the source resistance, rather than by $I_{s}$. This is because the initial current, not the possible source current, determines the arc current-time trajectory. We will discuss this later in more detail. Note that all the curves seem to be asymptotic to $I / I_{s}=1$.

Static characteristic curves, obtained by exercising the model with constant current sources for 20,50,80,100, and $200 \mathrm{~A}$, and for Thevenin equivalent sources, are shown in Fig. 7. The exercises were all started from the initial conditions as in Fig. 1. Values for the electric field were taken at elapsed model times of 10,30 , and $50 \mathrm{~ms}$. A similar process was followed for Thevenin source exercises; the load lines for these are displayed.

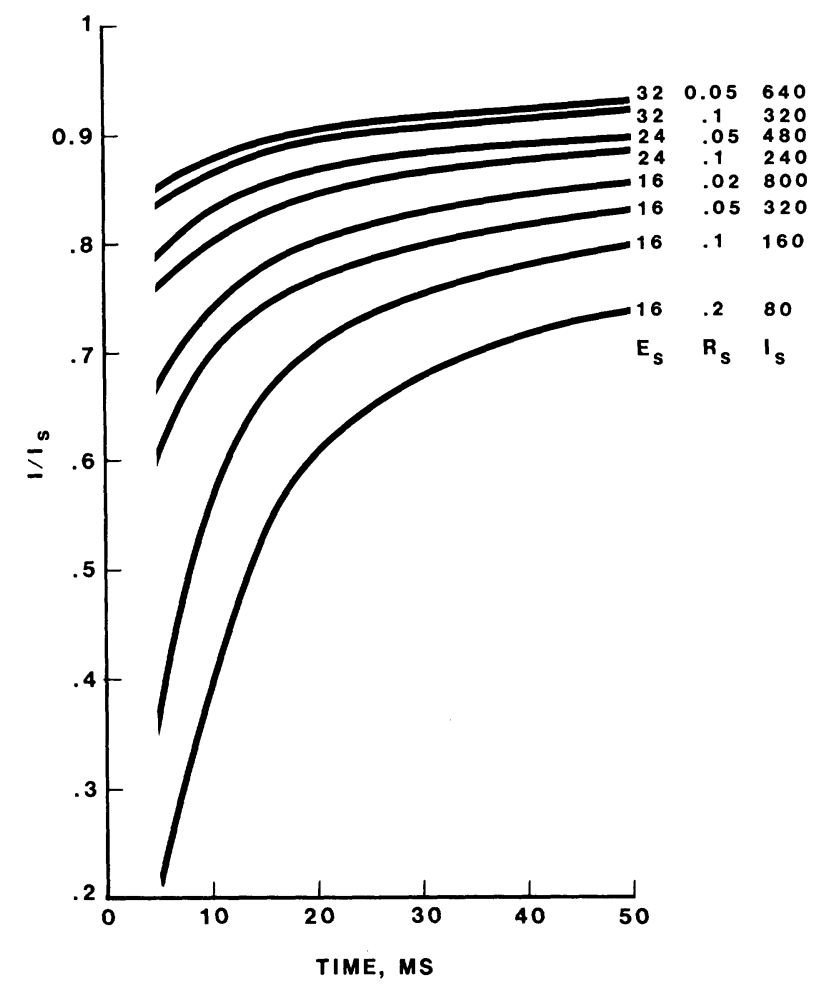

Fig. 6. Normalized current $I / I_{s}$ as a function of time for various Thevenin sources. (Units as in Fig. 2.)

The tendency for modeled arcs with Thevenin sources toward constant current behavior is again evident. The departure from constant current behavior becomes smaller with time, seen also in Fig. 6. In addition, as arc current increases for a given elapsed time, Thevenin channels approach constant current behavior. An attempt to fit the form $E=a+b I^{-n}$ ( $a, b$, and $n$ are constants, $E$ is the electric field on the arc, and I is arc current) showed a poor correspondence between that functional form, found to hold for some confined and short free arcs [16], and our model output.

Constant electric-field arc behavior was modeled by forcing the unit series resistance $R_{s}$ to be zero. Because the constant pressure assumption does not allow for sound or shock waves, most of the modeled arc currents became very large very quickly. That is, energy was available to the model channel so as to invalidate the assumptions. Channels with the same initial conditions but smaller constant field values cooled rapidly, with current rapidly decreasing to zero, that is the arc was not sustained. There is thus a field value that will produce a metastable state for each initial temperature distribution. To investigate this, the initial temperature profile was varied in radius. The metastable field value was found to be smaller as the initial size of the channel increased. We were able to approach this field value from both sides with respect to the size of the source field, and the rate of growth or decay of the model did slow as we got closer to the metastable field value. We could find, however, no simple means for establishing the metastable field value for a given initial temperature profile.

Except for some of the exercises with constant electric 


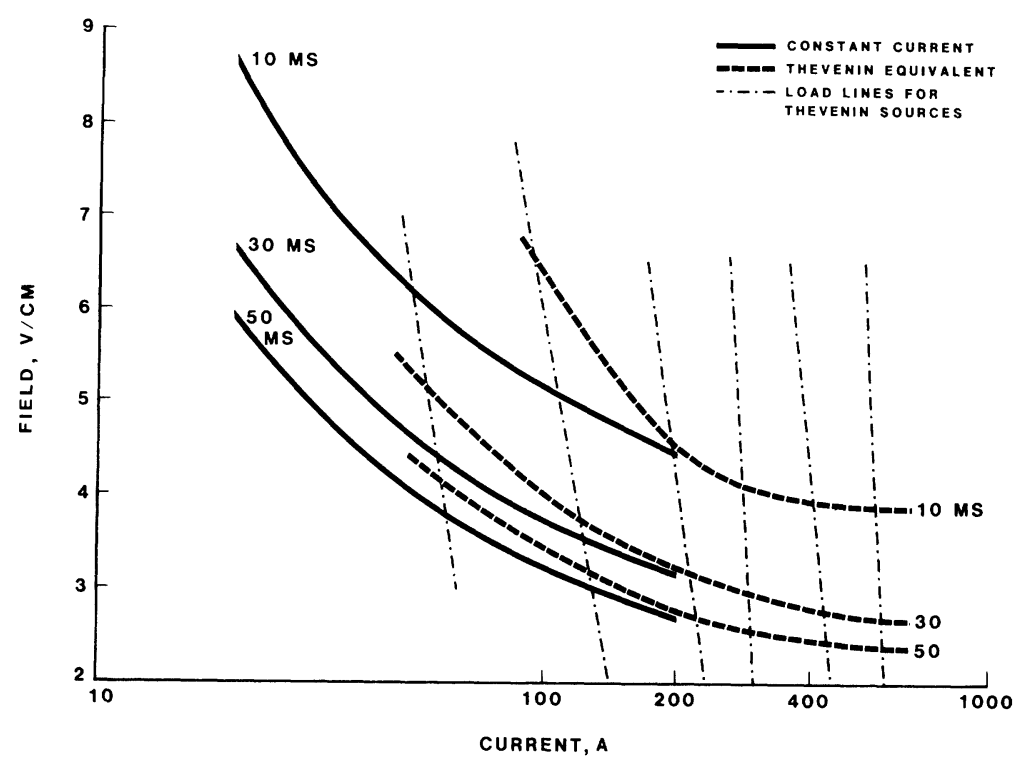

Fig. 7. Characteristic E - I curves for constant current and Thevenin sources.

field, the model resisted all attempts to generate a "hot core." An increase in current in the case of a constant current input, or an increase in field or decrease in source resistance in the case of a Thevenin source, resulted in temporary heating of the center portion of the arc. This increase was followed by a subsequent decrease much like the initial transient shown in Fig. 1, and the central temperature again decayed to the neighborhood of $6000 \mathrm{~K}$. The effect of the increases was thus to effectively restart the model at a larger radius, and on a different characteristic.

\section{The Characteristic Curve of the ArC}

The above results demonstrate that there is no unique characteristic curve of field versus current for the very long free vertical arc, and that the details of the radial expansion of the arc depend on the parameters of the electrical source in a complex way.

Suppose a model arc with a Thevenin source has been established and is expanding radially, in accord with the examples shown in the above figures. The operating point of the arc moves along the dimensionless load line, shown in Fig. 8, toward the constant current axis. That is, the current in the circuit is steadily increasing, and the field on the arc segment decreasing, according to the load line. As the arc channel expands radially, its resistance becomes smaller in comparison to the source, and the behavior closer to a constant current arc.

The center of the arc is slowly cooling, even though most of the energy supplied by the source is in this region. Radiation loss, although perhaps small, is not negligible, and can be responsible for much of the energy loss near the center of the arc. The rate of expansion depends on the "distance along" the load line (Fig. 8) toward the short-circuit current point $I / I_{s}=1$. The temperature in the center is usually between 6000 and $7000 \mathrm{~K}$.

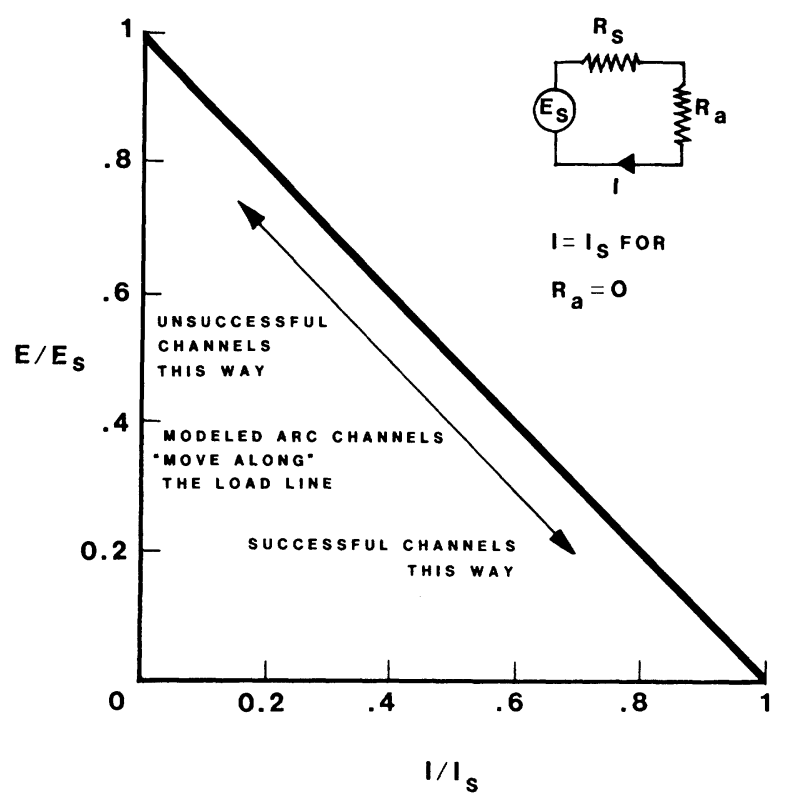

Fig. 8. Nondimensional load line showing trends for model arc load line trajectories.

The closer the operating point gets to the short-circuit current, the slower it approaches that value (Fig. 6). This is in contrast to the confined arc, which has, according to its length, electrode materials, and confining boundary, a fixed operating point on a given load line, unless the arc cannot be sustained by that source.

Now, in accord with the "classic" method of arc analysis, let the source be shifted, either to a higher field or to a lower unit resistance. The channel will respond by increasing in temperature near the center according to the local conductivity, on a time scale corresponding to that for confined arcs, on the order of hundreds of microseconds. Following this adjustment, unlike confined arcs, the channel begins to expand more rapidly than it had been 


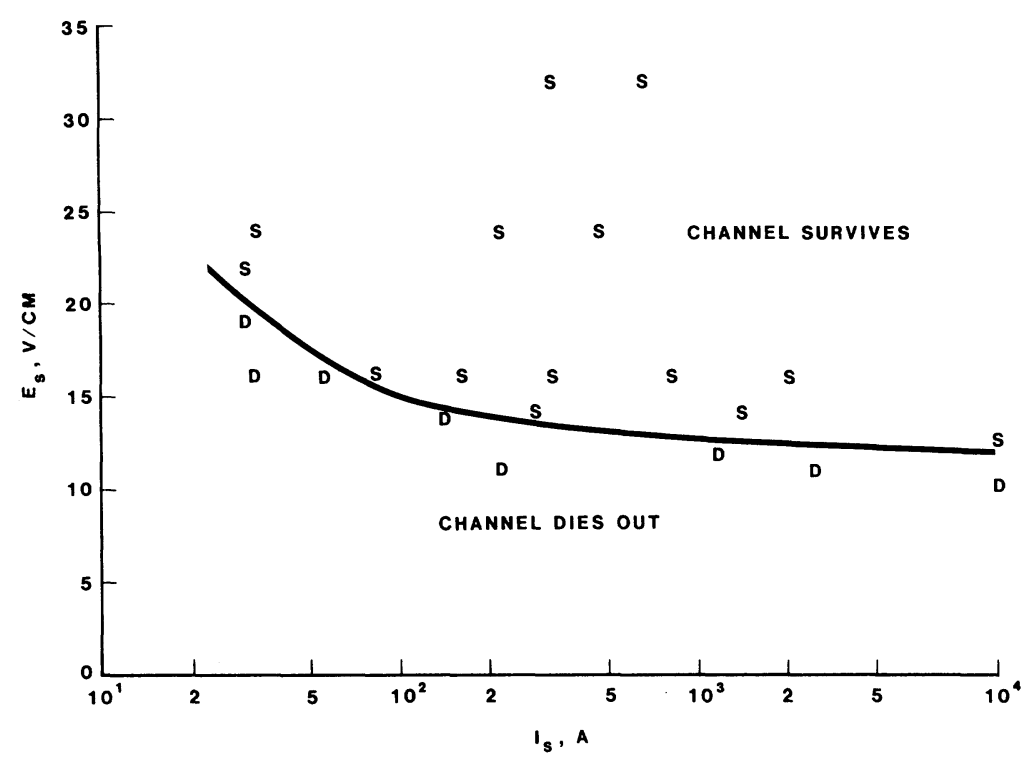

Fig. 9. Arc channel "survival", characteristics.

on a time scale of tens of milliseconds. The expansion and increased radiation cause the central zone temperature to fall, and the quasi-steady equilibrium to be established as before. The channel central temperature returns to the region of $6000-7000 \mathrm{~K}$.

If, on the other hand, the source field is reduced, or the unit source resistance increased, the temperature in the channel will tend to fall, again according to the local conductivity. The channel cannot contract; expansion of the outer edge must continue. If the field reduction or resistance increase is large enough, the arc will not be sustained, but the rate of temperature decrease in the center will be somewhat smaller than if the source had been interrupted.

The above analysis implies that the characteristic of a long arc with sinusoidal ac source should depend not only on the source voltage and impedance, but upon its frequency as well. Because the arc cannot sustain a "hot core" for low frequencies (say $100 \mathrm{~Hz}$ and below), it will expand as the current increases on the rising voltage of each half-cycle. On the falling half-cycle, the arc cannot contract. It can only expand more slowly, accompanied by a slow temperature decrease in the central portion of the arc. As the frequency is raised, however, the channel expansion will not take place as rapidly with respect to the driving frequency, and the arc may be able to generate a "hot core." In this way a high-frequency arc might actually expand more slowly than a low-frequency arc for the same rms source voltage.

Fig. 8 also shows that sources which might sustain a confined arc cannot sustain a free arc, since the arc operating point will move along the load line until the temperature in the central portion drops low enough to cause the conductivity to become small, and the current to decrease very rapidly. Fig. 9 shows a dividing line between sources that will and will not sustain an arc with the initial profile of Fig. 1.

\section{Conclusion}

The correspondence of our model results with the model in [3] is very good. The only experimental data we could find that met the conditions we have assumed, particularly with respect to length, are the measurements of Strom [2] and King [3], [17]. Although the latter reports a field value of about $10 \mathrm{~V} / \mathrm{cm}$ for the long vertical arc in [3], the background document for [3], i.e., [17], indicates that "in many cases the original results showed a large degree of scatter, on occasions as much as 4 to 1" [17]. No scatter or error analysis is included in either report. King's results are difficult to interpret because there is insufficient detail to permit us to ascertain whether our model assumptions are met in his experiments. Certainly both his results and Strom's do indicate that our model electricfield values are too low by a factor of 2 or so. Fig. 7 shows, however, that it is possible to obtain a large scatter in a volt-ampere characteristic measurement if the voltage and current observations are not made at the same time after arc initiation or if the initiation method for the arc is not the same from trial to trial. Furthermore, for arc currents between 20 and $200 \mathrm{~A}$, the source characteristics play a role in the arc characteristic obtained.

Strom's data as reported in [2] support our model predictions, at least qualitatively. Arcs whose first half-cycle current ratio $\left(I / I_{s}\right)$ was below 0.2 tended to extinguish in one or two cycles, whereas those with higher first halfcycle ratios tended to last for five to seven half-cycles. In addition, the first half-cycle value for successful arcs tended to be lower than the maximum current ratio indicating the kind of growth shown in Fig. 6. Strom's data give a range of $10-20 \mathrm{~V} / \mathrm{cm}$ for field values for his long arcs. He apparently did not compensate for tortuosity (which was at times considerable) in calculation of his reported field values.

The free boundary condition of the Appendix is correct for the free arc, and the moving wall is not. The difference 
is that because the heat transport occurs both by conduction and by mass transport, the velocity of a cold wall will not be correct; the moving wall does not properly conserve mass. Trials with our model show that even a boundary at a seemingly large distance from the arc has an effect on the model behavior.

The "time-constant" approximation, apparently useful for confined and/or short free arcs (e.g., [5]), may not prove a useful concept for the unbounded long arc. There are apparently two time constants involved, that for establishment of thermal equilibrium within the arc core, and another, on the order of 100 times longer, for establishing the growth demanded by the free boundary.

Future modeling effort will be directed toward the inclusion of self-magnetic effects in as simple a formulation as possible. We would like to see if these effects become important to free-arc behavior at the maximum current levels (about $1000 \mathrm{~A}$ ) in which we are interested. A rough estimate of the importance of magnetic field can be made in a couple of ways. First, we can calculate the radial magnetic pressure. For a $1-\mathrm{cm}$ radius channel and $100 \mathrm{~A}$ of current, this pressure is $\cong 1.6 \times 10^{-5} \mathrm{~atm}$. At 1000 $\mathrm{A}$, it would be $\cong 1.6 \times 10^{-3} \mathrm{~atm}$. Second, we can contrast the collision frequency $\nu$ against the cyclotron frequency $\omega_{c}$. For a 100-A 1-cm-diameter arc with a core temperature near $6000 \mathrm{~K}$, this ratio $\omega_{c} / \nu$ is about 0.05 , and for $1000 \mathrm{~A}$, it is 0.5 . These numbers indicate that the magnetic field might be significant.

We would also like to see if a technique such as that of Lee [8], can be used for a free boundary problem, so the long computation times of our model can be avoided. Certainly the boundary problem requires further examination (see Appendix) for asymptotic behavior. We should investigate the radiation behavior beyond the experiments of Schreiber et al. [13].

In addition, an arc generator has been built with which we may be able to test the models, and observation of the lightning continuing current, a naturally occurring very long arc, continues. We will try to look at the radiation from these real arcs together with that projected for the model to see if the combination can be used as a tool for examining the behavior of lightning continuing currents.

\section{APPENDIX \\ Model Equations and Computational Procedure}

The assumptions given in Section II as well as [4] result in the mass conservation equation

$$
\frac{\partial \rho}{\partial t}+\frac{1}{r} \frac{\partial}{\partial r}(\rho r u)=0
$$

the energy conservation equation

$$
\rho c_{p} \frac{\partial T}{\partial t}+\rho c_{p} u \frac{\partial T}{\partial r}=\frac{1}{r} \frac{\partial}{\partial r}\left(r k \frac{\partial T}{\partial r}\right)+\sigma E^{2}-Q
$$

the equation of state

$$
p=\rho R T Z
$$

and Ohm's law

$$
j=\sigma E
$$

as the model equations.

Here, $T$ is temperature, $\rho$ density, $C_{p}$ specific heat at constant pressure, $k$ thermal conductivity, $Z$ compressibility, $p$ pressure, $R$ gas constant, $\sigma$ electrical conductivity, $E$ electric field, $j$ current density, $Q$ radiation, $u$ radial velocity, $r$ radius, and $t$ time.

Rewriting (3) and integrating (1) gives

$$
\frac{\delta T}{\partial r}=-u \frac{\partial T}{\partial r}+\frac{1}{\rho r C_{p}}\left[\frac{\partial}{\partial r}\left(r k \frac{\partial T}{\partial r}\right)\right]+\frac{\sigma}{\rho C_{p}} E^{2}-\frac{Q}{\rho C_{p}}
$$

and

$$
u=-\frac{1}{\rho r} \frac{\partial}{\partial t} \int_{0}^{r} \rho r^{\prime} d r^{\prime} .
$$

Boundary conditions are specified as $r=0, \partial T / \partial r=0$ and $r \rightarrow \infty, T \rightarrow T_{a}$, the ambient temperature. Initial conditions are specified by giving an initial profile $T(r)$ at $t$ $=0$.

Diffusion similarity solutions, even with simplified equations for large radius and long time, do not apply. Attempts to solve the equations analytically, even with simplifying assumptions such as balance between joule heating and radiation for high currents, or neglecting radiation for low currents, have not been fully successful for either free or bounded arcs; transport property, radiation, and electrical conductivity functions for hot air are extremely complex, and the equations highly nonlinear.

Difference equations were formed from (5) and (6) as suggested in [18], and solved by an explicit numerical procedure. At each time step, a radial integration is performed on the right-hand side of the difference forms of (5) and (6). The last value and current value of the temperature are used to calculate the density in the difference form of (6). The temperatures are updated at the end of each radial integration. At the end of each time step, the total conductivity of the channel is available. This conductivity is used together with the source characteristics and (4) to calculate the value of $E$ for the next time step. Further details may be found in [4].

Our earlier model simulated the free outer boundary as suggested by Finklenburg [11]. The boundary was treated as a cold wall moving outward, with zero mass flow at the wall. The outward mass flow velocity is thus constrained to be the thermal diffusion velocity near this boundary, and the equations are not properly satisfied. Proper calculation of the free boundary requires that the model space radius be very large compared to the radius of the arc. A large part of calculation time is thus wasted.

The model used in this paper reduces the calculation time by using a diffusion boundary condition obtained by analytic solution of simplified arc equations. The conditions for the solution reduce the complexity of the equations by restricting the temperature to those values for 
which the ideal gas laws apply, the thermal conductivity and specific heat at constant pressure are substantially constant, and the electrical conductivity and radiation terms in the energy balance equation are negligible. The assumptions of these further restrictions give

$$
\rho C_{p}\left(\frac{\partial}{\partial t}+u \frac{\partial}{\partial r}\right) T=\frac{1}{r} \frac{\partial}{\partial r}\left(r k \frac{\partial T}{\partial r}\right)
$$

and

$$
\rho T=\rho_{a} T_{a} .
$$

$T_{a}$ and $\rho_{a}$ are the temperature and density at ambient conditions, taken here to be $300 \mathrm{~K}$. The conservation of mass equation is unchanged. Equations (7) and (8) hold quite well for temperatures below $1000 \mathrm{~K}$.

Combining these equations, we find that

$$
\frac{\partial}{\partial r}\left[r \frac{\alpha}{T_{a}} \cdot \frac{\partial T}{\partial r}-u r\right]=0
$$

where $\alpha$ is the thermal diffusivity at $T_{a}$. Equation (9) implies that the quantity in brackets is a function of time only for all radii meeting the assumptions of the derivation. Note that the range of radii which meet the criteria can, and does, change with time. If we choose two such radii, say $r_{1}$ and $r_{2}$, then we may write

$$
r_{1}\left[\left.\frac{\partial T}{\partial r}\right|_{1}-\frac{T_{a}}{\alpha} u_{1}\right]=r_{2}\left[\left.\frac{\partial T}{\partial r}\right|_{2}-\frac{T_{a}}{\alpha}\right] u_{2} .
$$

This relationship holds for two successive radii of a numerical approximation, and the result of carrying this out is

$$
\begin{aligned}
T_{n+1}= & T_{n}+\frac{H T_{a}}{\alpha} u_{n}+\frac{n-2}{n-1} \\
& \cdot\left[T_{n}-T_{n-1}-\frac{H T_{a}}{\alpha} u_{n-1}\right] .
\end{aligned}
$$

The radial step interval is $H$ and the radial step for which the temperature is to be calculated is $(n+1)$. This is the boundary condition applied to the model used in this paper; it is invoked during the radial integration at the first step for which the temperature is within $5 \mathrm{~K}$ of $T_{a}$.

The boundary formulation was checked against an identical model with a very large number of radial steps so that most of the model space was at ambient temperature and against the results in [3]. Both checks proved the difference equations and this method of boundary truncation satisfactory.

\section{ACKNOWLEDGMENT}

The author is indebted to Dr. F. Albini for many fruitful discussions. Thanks also to Dr. M. Uman and other reviewers for their comments.

\section{REFERENCES}

[1] L. A. King, "The voltage gradient of the free burning arc in air or nitrogen," in Proc. 5th. Int. Conf. Ionization Phenomena in Gases, H. Maecker, Ed. Amsterdam: North-Holland, 1962, pp. 871-877.

[2] A. P. Strom, "Long 60-cycle arcs in air," AIEE Trans., vol. 65, pp. 113-117, 1946.

[3] M. A. Uman and R. E. Voshall, "Time interval between lightning strokes and the initiation of dart leaders," J. Geophys. Res., vol. 73, pp. 497-506, 1968.

[4] D. J. Latham, "A channel model for long arc in air," Phys. Fluids, vol. 23 , no. 8, pp. 1710-1715, 1980.

[5] T. Matsumura et al., "Response of a wall-stabilized air arc to step and sinusoidal change in current," IEEE Trans. Plasma Sci., vol. PS8, no. 3, pp. 248-252, 1980.

[6] W. Hermann, "Computation of decaying wall-stabilized arcs," $Z$. Naturforsch., vol. 28a, pp. 443-453, 1973.

C. W. Kimblin and H. Edels, "Electrical conductance decay of interrupted arc columns," Brit. J. Appl. Phys., vol. 17, pp. 1607-1619, 1966.

[7] J. J. Lowke, "Characteristics of radiation-dominated electric arcs," J. Appl. Phys., vol. 41, no. 6, pp. 2588-2600, 1970.

[8] H. E. Lee, "'Solutions of plasma arc equations by the orthogonal collocation method," J. Phys., vol. 16, pp. 2191-2204, 1983.

[9] G. R. Jones and G. H. Freeman, "The influence of natural radial convention upon the transient behavior of a cylindrical arc column," Z. Phys., vol. 229, pp. 177-191, 1969.

[10] H. H. Maecker, "Principles of arc motion and displacement," Proc. IEEE, vol. 59, no. 4, pp. 439-449, 1971.

[11] W. Finklenburg, "The physical mechanism of low- and high-current arcs, and their relation to the welding arc," AIEE Trans., vol. 70, pp. 800-803, 1951.

[12] P. Adams, "High-temperature, equilibrium thermodynamic properties of chemically reacting mixture of $\mathrm{N}_{2}, \mathrm{O}_{2}, \mathrm{CO}_{2}, \mathrm{~A}$, and $\mathrm{Ne}$," Hughes Corp., Los Angeles, CA, Aerospace Tech. Res. Rep. 9, July 1966.

[13] P. W. Schreiber et al., "Electrical conductivity and total emission coefficient of air plasma," AIAA J., vol. 11, no. 6, pp. 815-821, 1973.

[14] W. Finklenburg and H. Maecker, Handbuch der Physik, V. XXII. Berlin: Springer-Verlag, 1956, p. 383.

[15] R. Breene et al., "Radiance of species and high temperature air," General Electric Corp., Schenectady, NY, Rep. R625D52, July 1962.

[16] J. Cobine, Gaseous Conductors. New York: Dover, 1958.

[17] L. A. King, "The voltage gradient of the free-burning arc in air or nitrogen," British Elec. and Allied Industries Res. Assoc., Surrey, England, Rep. G/XT172, 1961.

[18] H. S. Carslaw and J. C. Jaeger, Conduction of Heat in Solids, 2nd ed. Oxford, England: Oxford Univ. Press, 1959. 